Meta

Journal des traducteurs

Translators' Journal

\title{
Le traducteur et la documentation juridique
}

\section{Jean-Claude Gémar}

Volume 25, numéro 1, mars 1980

La documentation

URI : https://id.erudit.org/iderudit/002839ar

DOI : https://doi.org/10.7202/002839ar

Aller au sommaire du numéro

Éditeur(s)

Les Presses de l'Université de Montréal

ISSN

0026-0452 (imprimé)

1492-1421 (numérique)

Découvrir la revue

Citer cet article

Gémar, J.-C. (1980). Le traducteur et la documentation juridique. Meta, 25(1),

134-151. https://doi.org/10.7202/002839ar d'utilisation que vous pouvez consulter en ligne.

https://apropos.erudit.org/fr/usagers/politique-dutilisation/ 


\title{
Le traducteur et la documentation juridique
}

\author{
Jean-Claude GÉMAR \\ professeur, \\ Université de Montréal
}

La première difficulté, sans doute une des plus grandes, que le traducteur et le terminologue doivent affronter, durant leurs années de formation comme dans l'exercice de leur profession, leur est posée par la documentation.

La recherche du support documentaire qui fournira l'information notionnelle ou terminologique voulue pose un certain nombre de problèmes. Il faut ici faire le départ entre traduction «générale» et traduction «technique», celle de textes à caractère juridique étant entendue dans ce dernier sens.

S'il est un domaine où la question de la documentation revêt une importance particulière, c'est celui de la traduction juridique, en raison notamment du caractère normatif du droit. Avant de présenter la marche documentaire du traducteur nous analyserons les mécanismes généraux de l'économie de la documentation juridique.

\section{I. ÉCONOMIE GÉNÉRALE DE LA DOCUMENTATION JURIDIQUE}

Contrairement aux traducteurs spécialistes des questions d'électricité, de physique nucléaire ou de mécanique, le traducteur appelé à travailler dans le domaine juridique doit résoudre un problème inhérent à sa spécialité, soit rendre dans la langue d'arrivée spécialisée (L.S. ${ }_{2}$ ) des termes et des notions propres à la langue de départ spécialisée ( $\left.\mathrm{LS}_{1}\right)$ qui ne reposent pas toujours sur un dénominateur commun aux deux systèmes.

Bien que le langage de la physique, de la mécanique ou de l'électricité varic d'un pays à l'autre, il décrit des phénomènes somme toute identiques parce que les lois qui les régissent ignorent les frontières.

Tel n'est pas le cas de la règle de droit, dont l'application dépasse rarement le cadre limité des États ${ }^{1}$. C'est que l'objet du droit souffre nombre de définitions et que sa fin est fonction du type de système en vigueur et de la philosophie qui l'inspire. Il en découle une relativité des concepts particulièrement déroutante pour le traducteur, toujours en quête de certitude, qui travaille à cheval sur deux systèmes reposant sur un édifice social et politique dont

1. Il s'agit de la règle de droit interne et non de la règle de droit international, laquelle est censée s'imposer aux ordres juridiques étatiques, selon les tenants de la théorie moniste, dont Kelsen. 
l'architecture répond à des principes, des traditions et des aspirations spécifiques. L'édifice juridique qu'une nation a érigé est rarement semblable à celui d'une autre nation, même lorsque les origines de leurs systèmes juridiques sont plus ou moins communes.

Le traducteur juridique doit franchir les nombreux obstacles, dont les plus ardus ne sont pas toujours linguistiques, que présente l'opération de transfert. Aussi la documentation joue-t-elle un rôle primordial en traduction juridique. Nous allons tenter de définir la nature de la documentation avant d'étudier le rôle qu'elle joue en traduction, et de tracer les limites de son intervention.

\section{Section 1. Nature de la documentation juridique}

Le terme « documentation» connaît de nos jours une fortune qu'il faut probablement relier au développement foudroyant des techniques et des moyens d'information audiovisuels. L'essentiel $\mathrm{du}$ fonds documentaire n'en reste pas moins l'écrit, dont la prolifération effrénée ${ }^{2}$ menace de submerger ceux-là mêmes à qui elle est destinée. En outre, cette documentation pléthorique n'atteint pas pour autant son but comme sembleraient l'indiquer les résultats d'un sondage récemment effectué aux États-Unis ${ }^{3}$. L'adage nemo censetur legem ignorare (nul n'est sensé ignorer la loi) n'a plus sa raison d'être dans le contexte d'une société dont la complexité juridique n'est pas le moindre défaut.

Avant tout, il faut s'entendre sur le sens à donner à «documentation » car ce terme recouvre plusieurs réalités dans le domaine du droit, selon les systèmes et les pays considérés.

\section{Spécificitê de la documentation juridique}

Un traducteur exerçant son activité à Genève, à Paris ou à Rome et traduisant dans sa langue maternelle des textes juridiques anglais est placé dans une situation très différente de celle que connaît son homologue canadien. $\mathrm{Ce}$ dernier doit non seulement travailler en contexte de bilinguisme plus ou moins actif, ce qui n'a rien d'original en soi, beaucoup de pays étant dans le même cas, mais passer en outre fréquemment d'un système juridique à un autre, compte tenu du caractère hybride du droit canadien (notamment au Québec ${ }^{4}$ ), selon la nature du texte (droit public ou droit privé). Le Québec est placé à la croisée des deux grandes sources du droit canadien, la common law et le droit civil. Il faut $\mathrm{y}$ ajouter l'influence croissante du droit américain auquel se réfèrent, pour des raisons historiques, géographiques et linguistiques, les juristes canadiens.

Le rôle de la documentation, dans un tel contexte, est essentiel. La recherche documentaire passe souvent par trois ou quatre étapes, ce qui com-

2. Selon une estimation de l'American Bar Association, les seuls Etats-Unis publieraient chaque année 100000 ouvrages juridiques. Les textes de lois, règlements et décisions de justice se chiffrent par dizaines de milliers tous les ans.

3. American Bar Association Journal, June 1979 , vol. 65, p. 857.

4. Le droit public canadien (droit administratif, droit pénal, droit constitutionnel...) se rattache historiquement au droit public anglo-américain. Voir sur cette question : Henri Brun et Guy Tremblay, Droit public fondamental, Québec, Presses de l'Université Laval, 1972, p. 11-12 et 21-22; René Dussault, Traité de droit administratif canadien et québécois, Québec, P.U.L., 1974, t. 1, p. 14 et suiv. 
plique la tâche du traducteur en quête de sources fiables. Bien que ses préoccupations soient souvent fort éloignées de celles du praticien du droit qui connaît, en principe, le domaine et sa terminologie, elles ne se rejoignent pas moins sur la marche à suivre en matière de recherche documentaire : partir des ressources propres au domaine concerné. À cet égard, le droit est une des branches de l'activité humaine qui a produit le volume le plus considérable d'informations. Cette masse documentaire est aussi riche que complexe et variée. Toutefois, sa particularité procède du caractère normatif $\mathrm{du}$ droit ${ }^{5}$. Ce trait distinctif confère à la documentation juridique un degré de spécificité que l'on ne retrouve dans aucune autre science humaine.

Deux constatations en découlent : le traducteur et le terminologue ne devraient fonder leur recherche de l'information juridique que sur des éléments à valeur normative, seuls à même de leur apporter la bonne réponse; toute autre source d'information «non normative» doit être utilisée avec la plus grande circonspection. Avant d'établir une typologie des sources documentaires, on doit prendre en compte le facteur normatif. Autrement dit, le traducteur doit coller à la réalité du système juridique, quel qu'il soit.

\section{Typologie des sources de documentation en droit}

La typologie des sources du droit varie selon les systèmes juridiques. Dans le contexte nord-américain cependant, un certain consensus s'est formé autour des deux grandes catégories que sont la législation et la jurisprudence ${ }^{6}$. On distingue une troisième grande catégorie sous laquelle se rangent toutes les sources secondaires du droit : coutume, doctrine, etc.

\section{Sous-section 1. Les sources normatives}

Le caractère normatif du droit découle essentiellement de la législation et de la jurisprudence qui confèrent à la règle de droit sa validité, donc son efficacité et sa nature impérative, conditions sans lesquelles on ne pourrait parler de norme juridique.

\section{A. La législation}

La plupart des juristes nord-américains, qu'ils soient formés à la common law ou au droit civil, s'entendent pour reconnaitre la primauté actuelle de la législation comme source normative du droit, quelle que soit la forme sous laquelle elle se présente, lois, règlements d'application, décrets, etc. "

5. Voir sur cette question l'article de l'auteur, numéro spécial de Meta (vol. 24, no 1, mars 1979) consacré à la traduction juridique, p. 37-38; André Dunes, Documentation juridique, Paris, Dalloz, coll. \&Méthodes du droit \$, 1977, no 13, p. 10.

6. Il n'est pas dans notre intention, dans le cadre limité du présent article, d'entrer dans le débat opposant théoriciens et praticiens du droit sur la valeur relative de telle source par rapport à telle autre. L'ordre juridique se fonde généralement sur la constitution, loi suprême d'un pays. Son étude relève plutôt de la science politique.

7. En common law, voir en particulier F.A.R. Chapman, Fundamentals of Canadian Law, Toronto, McGraw-Hill of Canada, 1965, p. 13-14; Gerald L. Gall, The Canadian Legal System, Toronto, Carswell, 1977, p. 23-24. En droit civil, voir notamment Denis Le May, Méthode de recherche en droit québécois et canadien, Québec, P.U.L., 1974, Introduction, IV. 
La norme fixée par le législateur s'impose au traducteur à qui il n'appartient pas de choisir entre plusieurs termes celui qui semble le mieux convenir à la situation. La loi est au-dessus du contexte et des considérations linguistiques. On peut contester l'emploi du mot «corporation» et lui préférer celui de «société commerciale». Il reste que le terme défini dans la loi est celui que le juge, soucieux de respecter l'unité et la stabilité sémantique nécessaires au bon fonctionnement de la machine judiciaire, retiendra en fin de compte.

La loi est la source première d'information du traducteur, elle n'est pas la seule. La jurisprudence est un autre aspect majeur de l'impératif juridique qui s'exprime par la règle de droit.

\section{B. La jurisprudence}

L'important rôle supplétif de la législation que joue la jurisprudence nordaméricaine porterait certains observateurs à la placer au-dessus de la loi comme source normative du droit. On sait la place qu'occupe la jurisprudence en droit anglo-américain. Historiquement, la common law est un droit d'essence jurisprudentielle. Bien que le principe du stare decisis ne soit pas suivi de la même manière dans tous les pays de langue anglaise, il ne s'en dégage pas moins une autorité de la chose jugée qui est sans équivalent dans les pays de tradition romano-germanique. Aussi la jurisprudence, en régime de common law, occupe-telle une place à part et sa valeur normative est-elle grande. La nature et les fonctions de la Cour suprême, au Canada comme aux États-Unis, témoignent du rôle privilégié $\mathrm{du}$ juge dans une société justement appelée «de droit ${ }^{8}$.

\section{Sous-section 2. Les sources secondaires}

Par secondaire, il ne faut pas entendre négligeable. Les sources secondaires du droit sont celles dont le caractère contraignant (ou normatif) est moindre que celui de la législation ou de la jurisprudence. D'ailleurs, en règle générale, peut-on affirmer que la coutume est « secondaire» par rapport à la loi ? Certains pays, et non des moindres, font à la coutume, à la tradition une place qu'elle n'occupe pas en régime de common law. Pour les besoins du présent article, nos observations se limitent aux deux principaux systèmes juridiques du monde occidental : common law et droit civil. En outre, nous prenons en compte des éléments documentaires qui, pour le juriste, ont une valeur normative très faible, voire nulle. Pour le traducteur, en revanche, les données recueillies dans un dictionnaire ou un lexique sont précieuses.

Parmi les sources de documentation dans lesquelles traducteurs et terminologues puiseront leurs renseignements, il faut citer d'une part la coutume, la doctrine, les répertoires, encyclopédies, dictionnaires, lexiques et autres vocabulaires, uni-, bi- et multilingues, et d'autre part, tout ce que l'on peut ranger sous la rubrique « divers », c'est-à-dire les formulaires, modèles (contrat type par ex.),

8. Sur la question de la jurisprudence et de l'organisation judiciaire aux Etats-Unis, voir René David, Les grands systèmes de droit contemporains, 6e éd., Paris, Dalloz, p. 435 et suiv. 
rapports (Commission de réforme du droit du Canada, Commission de réforme du Code civil...), conventions collectives, etc.

\section{A. La coutume}

Bien que la coutume dispute encore la première place à la loi dans de nombreux pays, elle occupe une place secondaire dans la hiérarchie des sources documentaires du traducteur, pour qui l'ecrit et le contexte sont essentiels. C'est en général vers la doctrine qu'il se tourne pour aller chercher un complément d'information, un éclaircissement ou une définition.

\section{B. La doctrine}

Sous ce terme général se cache un nombre considérable d'écrits présentant un très grand intérêt pour le traducteur et le terminologue : tout ce qu'écrivent théoriciens et praticiens du droit (professeurs, hommes de loi, juges...) et qui présente un caractère didactique ou critique. Ce sont, pour l'essentiel, les ouvrages généraux et les manuels, les monographies et les thèses, d'une part; les articles de revues juridiques (sans exclure pour autant les revues générales et la presse), commentaires d'arrêt et commentaires législatifs d'autre part, ces derniers présentant moins d'intérêt pour le traducteur que pour le juriste.

C. Répertoires, encyclopêdies, dictionnaires...

Les mots " répertoire », "encyclopédie 》, "dictionnaire 》, etc. exercent une grande séduction sur le traducteur et le terminologue pour lesquels ils font sérieux, scientifique car ils ont vocation à l'exhaustivité, mythe particulièrement tenace chez le lexicographe, et, ce qui est encore plus grave, à la fiabilité.

Combattons au passage quelques idées reçues. Paul Doucet, l'éminent lexicographe juridique, pensait que l'on devait considérer comme bon et exhaustif le dictionnaire contenant de 85 à $90 \%$ des mots que l'on cherche ${ }^{9}$. Quant à leur fiabilité...

Cette source documentaire est néanmoins un élément d'orientation des plus utiles dans la recherche notionnelle ou terminologique.

\section{Autres sources documentaires}

Lorsque l'on fait une recherche, aucun élément n'est à négliger. Le droit est une discipline particulièrement riche en documents de toutes sortes. Le traducteur n'a souvent que l'embarras du choix entre les innombrables actes notariés, contrats, formulaires types, procès-verbaux d'audiences, etc., qui abondent en données informatives sur le style administratif ou judiciaire, le vocabulaire juridique, ou fournissent des contextes, des formules caractéristiques.

Après avoir défini les caractéristiques générales de la documentation juridique utile au traducteur, voyons quel en est le rôle.

9. Actes du Stage de Saint-Cloud (22-30 nov. 1967), Les langues de spécialité : analyse linguistique et recherche pédagogique, Strasbourg, AIDELA, 1970, 256 p. 


\section{Section 2. Les fonctions de la documentation juridique}

La documentation juridique remplit un certain nombre de fonctions qui guident le traducteur dans sa recherche du renseignement. Parmi toutes les fonctions possibles en théorie, étant donné la nature multidisciplinaire du droit ${ }^{10}$, il en est deux que la documentation juridique exerce particulièrement, soit la fonction informative et la fonction normative.

\section{Sous-section 1. La fonction d'information}

L'objet de la traduction étant la communication ${ }^{11}$, le traducteur doit fonder son message sur une information fiable, relativement complète et pertinente, qu'une recherche documentaire répondant à ces trois critères est en mesure de lui fournir.

De nos jours, la recherche et la consultation de la documentation juridique présentent un caractère fonctionnel marqué qui devrait en principe faciliter la tâche du traducteur et du terminologue. Les progrès remarquables accomplis sur les plans de la compilation des unités documentaires et de leur consultation découlent des techniques et des moyens informatiques. Les banques de mots sont un exemple que les terminologues ont su exploiter avec un certain succès. L'exemple de SOQUIJ et d'autres systèmes de diffusion de la jurisprudence (ou d'autres données juridiques ${ }^{12}$ ), pratiquement au jour le jour, est encore plus probant. Tout en rendant de grands services aux praticiens, ils constituent une aide précieuse pour les chercheurs. À cet égard, l'informatique est un facteur d'accélération dans la communication matérielle des données juridiques ${ }^{13}$. Elle permet de tendre vers une certaine exhaustivité et de livrer les renseignements « à chaud », dans la foulée de l'événement. Un tel traitement de l'information brute (intégrale, résumée ou sous forme de mots clés) laisse à l'utilisateur le soin de faire la sélection qu'il juge appropriée et ne garantit nullement la valeur «scientifique» des données transmises. La qualité de l'information n'est pas proportionnelle à la vitesse de sa communication. La part de l'étude et de la réflexion pondérées, de l'opération synthétique et non analytique pour replacer l'unité documentaire dans son contexte global, à la fois synchronique et diachronique, devrait être plus grande. Il y aura toujours place, dans la sélection idéale de la documentation juridique, pour des ouvrages comme Les grands arrêts de la jurisprudence... De même, dans un autre ordre d'idées, les codes

10. Un rapide coup d'œil sur les programmes des facultés de droit les plus réputées confirmera que l'economie, la sociologie, la philosophie, etc., font partie intégrante des études de droit.

11. Voir d'Eugene A. Nida, «Translating Means Communicating : A Sociolinguistic Theory of Translation 》, in Linguistics and Anthropology : Georgetown University Round Table 1977, Washington, D.C., Georgetown University Press, 1978.

12. La Société québécoise d'information juridique, constituée par la loi L.Q. 1975, chap. 12 , le $1 \mathrm{er}$ avril 1976, a pour fonctions, aux termes de l'art. 19, de " promouvoir la recherche, le traitement et le développement de l'information juridique en vue d'en améliorer la qualité et l'accessibilité au profit de la collectivité ». En France, l'Institut de recherches et d'études sur le traitement de l'information juridique et le Centre d'études et de traitement de l'information juridique (IRETIJ-CETIJ) jouent un rôle à peu près semblable.

13. Voir L'Information juridique : du rêve à l'instrument, Montréal, Thémis, $1975,391 \mathrm{p}$. 
de lois annotés et commentés par les spécialistes reconnus d'un domaine feront longtemps encore autorité.

Le traducteur doit, et cela fait partie des obligations de son métier, suivre l'évolution des méthodes documentaires dans le domaine de sa spécialité. Toutefois, sa quête de l'information est conditionnée par le facteur normatif, dont le caractère impératif dépend d'un nombre $x$ de variables qui sont fonction du système juridique considéré. Cette préoccupation et ces contraintes rendaient nécessaire l'établissement de bibliographies répondant aux besoins spécifiques du traducteur ainsi qu'aux critères de fonctionnement fixés par les juristes ${ }^{14}$.

\section{Sous-section 2. La fonction normative}

La fonction normative est l'élément fondamental de la documentation juridique, le fil conducteur qui doit guider la recherche, tout particulièrement lorsque le texte à traduire présente un intérêt public ${ }^{15}$ (législation par exemple) en raison du caractère impératif de la règle de droit, mais également dans le cas de textes d'intérêt privế ${ }^{16}$ puisque « le contrat est une convention par laquelle une ou plusieurs personnes s'obligent envers une ou plusieurs autres, à donner, à faire ou à ne pas faire quelque chose ${ }^{17}$.

La recherche documentaire, dans le cas de textes de droit public, devrait être théoriquement plus rigoureuse que pour les textes de droit privé puisque la loi est au sommet de la hiérarchie normative ${ }^{18}$, précédant les pouvoirs exécutif et judiciaire, et présente un intérêt général absent des conventions définies à l'article 1101 du Code civil. En réalité, ces deux types de textes sont difficilement comparables et leur degré de difficulté n'est pas proportionnel à la nature publique ou privée de leur objet.

La valeur normative du renseignement que le traducteur cherche à obtenir découle principalement des trois variables énoncées plus haut dont dépend la fiabilité de la source documentaire. Pour le traducteur, cette fiabilité est la qualité première d'un dictionnaire unilingue ou bilingue, support principal de son activité. Il est donc naturellement porté, à tort ou à raison, à lui accorder une grande importance, celle que l'artisan place dans l'instrument de son art. Or cette confiance n'est pas toujours bien placée, les productions lexicographiques, en droit, égalant rarement les grands dictionnaires généraux.

\section{Fiabilité des sources documentaires}

Le juriste, notamment le praticien qui ne fait pas profession de traduire, consulte peu les dictionnaires de droit. Cela s'explique par le fait qu'il connaît à la fois la matière et la terminologie, contrairement au traducteur dont la

14. En particulier, de l'auteur, Bibliographie sélective du traducteur - Droit et justice,

15. Montréal, Linguatech, 1978,300

15. Art. 10 du Code civil du Québec : \&Tout acte est public à moins qu'il n’ait été déclaré privé. Chacun est tenu de prendre connaissance des actes publics. »

16. Art. 1065 du Code civil du Québec : \& Toute obligation rend le débiteur passible de dommages en cas de contravention de sa part. »

17. Art. 1101 du Code civil, Paris, Dalloz.

18. Henri Brun et Guy Tremblay, Droit public fondamental, op. cit., p. 167-168. 
première étape de la recherche passe le plus souvent par le dictionnaire : à partir du mot il remonte à la source notionnelle. Aussi ce mot doit-il présenter un maximum de garantie. Prenons un exemple classique, celui de common law, et voyons les équivalents que proposent quatre dictionnaires de traduction, deux généraux (Robert et Collins, Harrap's Standard), et deux juridiques (Nouveau dictionnaire Quemner, Doucet) :

Robert et Collins : droit coutumier

\begin{tabular}{|c|c|c|}
\hline Harrap & : & $\begin{array}{l}\text { (i) le droit romain, le droit coutumier } \\
\text { (ii) le droit civil }\end{array}$ \\
\hline Quemner & : & $\begin{array}{l}\text { droit coutumier et droit jurisprudentiel } \\
\text { (suivi d'une explication diachronique) }\end{array}$ \\
\hline Doucet & : & ce terme n'y figure pas ! \\
\hline
\end{tabular}

Que faut-il en déduire? Aucune réponse n'est hélas acceptable dans le contexte canadien. Que l'on dise en France que la Common Law = droit « coutumier », « civil », « commun » ou « jurisprudentiel » ne porte pas à conséquence puisque ce système n'y revêt aucune valeur normative, que la rule of law ne s'y applique pas. On ne fait que perpétuer une illusion (sauf pour ce qui est de la nature jurisprudentielle de la Common Law), celle qui voudrait que la Common Law soit un droit «coutumier» non écrit, par opposition au droit «écrit» (sous-entendu, le système romano-germanique). Au Canada, c'est la (ou le) common law (sans italiques) qu'il convient de dire. Cette expression est attestée par la doctrine lorsque le contexte indique qu'il s'agit du «body of law borne out of British legal tradition and built up and developed over centuries of judicial pronouncements ${ }^{19}$ ». On pourrait ajouter avec F.A.R. Chapman, "Much of this law has been put into statute form by Parliament ${ }^{20}$ ». Et que l'on ne vienne plus nous parler de droit coutumier "non écrit \$ après cela ${ }^{21}$ !

Deuxième constatation, aucun des quatre dictionnaires ne fait la distinction entre le système juridique (common law) et l'ensemble des règles applicables en droit privé (droit commun), qui se dit également common law. Comment un traducteur néophyte peut-il s'y retrouver ? Nous touchons là une des faiblesses des dictionnaires, celle que comporte leur utilisation en dehors du cadre sociologique dans lequel ils ont été établis. Et cela vaut pour les dictionnaires généraux comme pour les spécialisés.

Afin de ne pas tomber dans les chausse-trappes que contient la traduction juridique, le traducteur débutant évitera de faire une trop grande confiance aux dictionnaires. Le facteur temps joue un rôle crucial en traduction. C'est donc à

19. Gerald L. Gall, The Canadian Legal System, op. cit., p. 1, Introduction. Voir aussi René David, Les grands systèmes de droit contemporains, op, cit., para. 266, p. 317 et suiv.; Henri Brun et Guy Tremblay, Droit public fondamental, op. cit., p. 25 et suiv.; René Dussault, Traité de droit administratif, op. cit., t. II, p. 1476-1477; Me LouisPhilippe Pigeon, Rédaction et interprétation des lois, notes du cours donné en 1965 aux conseillers juridiques du gouvernement du Québec, p. 6.

20. F.A.R. Chapman, Fundamentals of Canadian Law, op. cit., p. 13.

21. Sur cette importante distinction entre common law et customary law, voir Blackstone, Commentaries, Book I, p. 115, note 5. Customary Law, Baton Rouge, Claitor's, 1976; Radcliffe and Cross, The English Legal System, 6th ed., London, Butterworths, 1977, p. 21 et suiv. ( + index). 
l'université, sur les bancs des cours de traduction, que l'étudiant doit acquérir les principes de la méthode documentaire, dont les mécanismes, pour être efficaces, procèdent d'une logique quasi formelle.

\section{L'APPROCHE DOCUMENTATRE DU TRADUCTEUR JURIDIQUE}

Comme dans la plupart des disciplines, en traduction tout commence par la lecture attentive du texte. Est-il encore besoin d'insister sur cette phrase essentielle de l'opération de traduction? Sans doute, à voir le nombre encore trop grand d'étudiants (et même de traducteurs confirmés...) escamoter une étape jugée secondaire lorsqu'ils ont un travail à faire en temps limité.

C'est oublier trop vite que la phase de lecture, pour le traducteur, correspond en gros à celle du plan que doit préparer l'étudiant avant de faire une composition ou une dissertation. Faute de suivre le cheminement de la logique interne du texte, le traducteur peut trébucher sur l'obstacle pour n'avoir pas saisi toute la portée du contexte ${ }^{22}$. C'est le cas de ceux qui ont traduit «Law » par «La loi », dans la phrase suivante :

Law essentially serves two functions in modern, western, industrial society.

De tels exemples sont légion. Afin d'éviter semblable mésaventure, ou tout au moins de prendre le maximum de précautions pour éviter une erreur toujours possible, le traducteur de textes juridiques pourrait suivre la méthode que nous allons exposer.

\section{Cheminement de la recherche documentaire en traduction juridique}

L'opération documentaire du traducteur comprend trois étapes principales : lectures et analyse du texte; relevé des termes et notions inconnus; recherche des équivalents. La troisième étape est celle qui nous intéresse, aux fins de notre étude.

Supposons que le paragraphe qui suit fasse partie d'un texte que l'étudiant doit traduire chez lui, et qu'il dispose d'une semaine au moins pour ce faire.

The anticipatory repudiation

The early law recognized no such anomaly as the possibility of breach of contract before the time for performance is due. An anticipatory repudiation is no more than an announcement of intention by a promisor that in the future $[. .$.$] he will not render his performance.$

Handbook of the Law of Contracts, 2nd ed., St. Paul, Minn., West, 1965.

Comment procéder à partir de ces données? Une lecture attentive apportera trois types de renseignements : il s'agit d'un manuel traitant de Law of Contracts, publié aux États-Unis (et non en Angleterre), relativement récent.

\section{Les étapes de la recherche}

Le profane, ignorant des sources documentaires du droit, s'en remet aux dictionnaires de traduction pour trouver la solution. Or cette consultation devrait

22. Roda P. Roberts, "Context in Translation 》, in Actes du 10e colloque de l'Association canadienne de linguistique appliquée, mai 1979, particulièrement p. 127 et suiv. 
plutôt intervenir en dernier, lorsque l'on dispose de suffisamment de temps pour approfondir la question. Le relevé des mots clés les plus signifiants doit permettre de cerner de plus près l'objet du texte. Dans l'extrait proposé, breach, contract, performance, anticipatory, repudiation, etc. le mettront sur la bonne voie. La logique voulant que l'on aille du général au particulier, dans ce type de recherche, l'opération de recherche pourrait se présenter ainsi :

1) LAW OF CONTRACTS (thème général)

2) TERMINATION of contract (relation intermédiaire)

3) BREACH of contract (aspect particulier de 2)

4) ANTICIPATORY breach of contract (stade final, aspect le plus particulier)

Une recherche rapidement menée donnera un résultat « nécessaire». Le Black's Law Dict., sous la vedette BREACH OF CONTRACT, a une entrée pour Anticipatory Breach. Eurêka? Pas encore, car ce n'est pas suffisant. Ici s'arrêteront les recherches de la plupart des traducteurs. Certains de tenir la solution, ils se contenteront de vérifier la traduction de breach of contract dans un dictionnaire juridique bilingue et l'assortiront de l'adjectif «anticipé(e) 》. Ce faisant, ils continueront d'ignorer que 1) il n'existe pas, en droit français, de «droit des contrats» comme tel;2) la notion de « rupture anticipée de contrat $\gg$ n'existe pas non plus, en droit français.

Aussi l'étudiant consciencieux n'en restera-t-il pas là mais lira par exemple un manuel sur la question des contrats, voire l'arrêt fondamental en l'espèce, que signale Black.

Il est bon de saisir le plus de nuances possibles dans le cas d'une notion comme celle-ci, afin de les faire passer dans $\mathbf{L S}_{2}$. Une partie, importante certes, de la recherche a été accomplie mais il reste à faire la même opération dans la langue d'arrivée. Il est conseillé de suivre pour $\mathrm{LS}_{2}$ le cheminement suivi pour $\mathbf{L S}_{1}$. En droit français, le contrat fait partie de la théorie générale des obligations, dont il n'est qu'une des deux sources principales ${ }^{23}$. Il en découle qu'une recherche en «droit des contrats» passe obligatoirement par les obligations conventionnelles. En $\mathrm{LS}_{2}$, le cheminement suivrait donc ce schéma :

1) CONTRAT (terme général)

2) FIN du contrat (situation possible)

3) INEXÉCUTION du contrat (manière de mettre fin au contrat)

4) inexécution ANTICIPÉE ${ }^{24}$ (terme spécifique)

Quelle conclusion tirer de cette démarche? Une constatation s'impose : en traduction juridique, les dictionnaires bi- ou multilingues n'ont pas pour objet de proposer des solutions à tous les problèmes susceptibles de se poser. Le

23. Le Code civil distingue deux sortes d'obligations, les obligations « conventionnelles », qui naissent d'une convention, autrement dit d'un contrat (art. 1101 et suiv.), et les obligations qui se forment sans convention, soit sans contrat (art. 1370 et suiv.).

24. Sans doute le meilleur équivalent possible. Voir René David, Les contrats en droit anglais, Paris, L.G.D.J., 1973, p. 373. 
traducteur doit en conséquence se reposer sur d'autres sources documentaires, encore plus fiables. Tel est le cas de la doctrine qui joue un rôle de support fondamental correspondant à sa vocation d'analyse et de critique du droit (législation et jurisprudence). Les manuels de droit sont les auxiliaires indispensables du traducteur qui y trouvera les renseignements (notions, terminologie, contextes définitoires...) nécessaires pour parvenir à la solution acceptable en LS $_{2}$. Cela n'exclut pas le recours à d'autres moyens de vérification et de contrôle comme la consultation d'experts.

Le traducteur pourrait suivre le cheminement méthodologique suivant :

1. Lecture et analyse du texte

2. Relevé des mots clés pour trouver les vedettes-matières

3. Consultation des sources documentaires, en langue de départ (LD), et en langue d'arrivée (LA)

\begin{tabular}{|c|c|}
\hline LD & LA \\
\hline $\begin{array}{l}\text { 1. Recherche sommaire (les notions sont } \\
\text { connues du traducteur) } \\
\text { a) dict. ou encycl. jurid. unil. } \\
\text { 2. Recherche approfondie (notions mal } \\
\text { connues ou inconnues du traducteur) } \\
\text { a) dict. ou encycl. jurid. unilingue } \\
\text { b) doctrine (manuels de droit) ou juris- } \\
\text { prudence, ou les deux } \\
\text { c) autres sources (consult. d'experts par } \\
\text { ex.) }\end{array}$ & $\begin{array}{l}\text { 1. Recherche sommaire (notions connues } \\
\text { du traducteur) } \\
\text { a) dict. ou encycl. jurid. unilingue } \\
\text { b) dict. juridique de traduction } \\
\text { 2. Recherche approfondie (notions mal } \\
\text { connues ou inconnues du traducteur) } \\
\text { a) dict. ou encycl. jurid. unilingue } \\
\text { b) doctrine (manuels de droit) ou jurisp., } \\
\text { ou les deux } \\
\text { c) dict. jurid. de traduction } \\
\text { d) autres sources } \\
\text { e) comparaison et vérification }\end{array}$ \\
\hline
\end{tabular}

On voit la place qu'occupent doctrine et jurisprudence dans la recherche documentaire en droit. Le traducteur doit en tenir compte et ne pas se contenter d'une démarche ponctuelle mais rechercher une perspective globale à partir de laquelle, par élimination successive des données non pertinentes, il arrivera à la solution.

Auparavant, il lui faut compter sur une documentation fiable; relativement complète et... accessible.

\section{Le support documentaire du traducteur juridique}

Tous les traducteurs ne disposent pas des unités documentaires répondant à ces trois critères. La salle de documentation tient une grande place dans la profession de traducteur. Voyons quels éléments il devrait y trouver pour mener ses recherches à bien.

\section{La documentation personnelle}

À moins qu'il ne dispose d'un centre de documentation à proximité, le traducteur ne peut compter que sur ses ressources personnelles pour organiser 
sa propre documentation et tirer le meilleur parti de son investissement. Le support documentaire minimal peut être regroupé sous les quatre rubriques suivantes :

\section{- Bibliographies}

Toute étude le moindrement approfondie d'un sujet exige de la part du chercheur le recensement préalable des instruments documentaires particuliers au domaine considéré. Les bibliographies lui facilitent la tâche en présentant sous une forme condensée et pratique les références essentielles compilées par les spécialistes du domaine.

Deux types de bibliographies sont à recommander, les générales et les spécialisées. Les premières servent de point de départ à toute étude, celle du profane comme celle du spécialiste ; les secondes ont pour fonction d'introduire le chercheur dans le champ de plus en plus restreint du secteur étudié, selon le processus suivant :

1. bibliographie du droit (anglo-américain par ex.)

2. bibliographie du droit (commercial canadien)

3. bibliographie du droit d'auteur (au Canada)

Deux bonnes bibliographies générales devraient rendre les services attendus. Le choix des bibliographies spécialisées est fonction du système juridique, de l'État ou de la Province concernés. Une documentation commerciale française peut ne pas rendre les services escomptés au traducteur de Los Angeles, de Vancouver ou de Toronto. Et vice versa.

- Dictionnaires, encyclopédies, lexiques...

Les productions lexicographiques sont le principal outil de travail du traducteur, aussi est-il normal qu'il leur témoigne une estime particulière et se sente d'autant plus frustré lorsqu'elles ne répondent pas à son attente. Dans le secteur juridique, les bonnes productions, en plus d'être chères, sont rares. Le traducteur doit procéder à une évaluation précise de ses besoins et de ses moyens avant d'acquérir quelque dictionnaire que ce soit. Nous lui conseillons d'acquérir :

- deux dictionnaires unilingues anglais (Black et Osborn ou Jowitt, par ex.)

- deux dictionnaires unilingues français (Capitant et Lexique de termes juridiques)

- un ou deux dictionnaires juridiques généraux et bilingues (Quemner, Doucet)

- le(s) lexique(s), glossaire(s), etc. spécialisé(s), selon le domaine de spécialité.

Ces ressources lexicographiques ne devraient constituer qu'un fonds documentaire minimal. Il conviendrait d'y ajouter deux encyclopédies juridiques (une 
en anglais, une en français) sans compter les dictionnaires généraux dans les deux langues (Larousse, Robert, Webster ; Robert et Collins, Harrap). L'investissement est lourd mais il devrait être rentable.

- Codes, législation...

Conseiller au traducteur de se munir des divers codes en vigueur dans un pays donné peut paraître superflu car il sait tout l'intérêt que présentent ces recueils de lois, surtout lorsqu'ils comprennent un index des mots clés et des descripteurs, ce qui est généralement le cas. La consultation des index du Code criminel par exemple, permet de trouver certains équivalents qui ne figurent pas dans les dictionnaires.

Il est également bon, ainsi qu'il a déjà été dit, d'avoir dans sa bibliothèque les textes de la législation fédérale (ou provinciale) en vigueur dans son domaine de spécialité. Il s'agit de fascicules, généralement commodes et bon marché, qui seront très utiles lorsque le traducteur voudra employer les termes exacts de la loi et hésite sur la traduction d'expressions comme trust company ou moveable property.

\section{- La doctrine}

Ce titre général recouvre plusieurs catégories d'ouvrages de référence très utiles, qui vont des monographies aux manuels de droit, du «Que sais-je ? 》 au traité en plusieurs volumes, en passant par les périodiques. Signalons ici la grande place faite en droit aux références et aux abréviations, notamment lorsqu'il y a chevauchement des systèmes, comme au Canada. Le traducteur peut facilement s'égarer dans ce dédale... Nous lui conseillons de se documenter sur la question avant de se plonger dans une recherche ${ }^{25}$.

\section{a) Les ouvrages de référence}

Deux types d'ouvrages sont à conseiller : un bon manuel de droit comparé et quelques manuels d'introduction au droit (anglo-américain et français) et d'initiation $\mathrm{au}(\mathrm{x})$ domaine(s) de spécialité du traducteur.

C'est dans un manuel de droit comparé que celui-ci trouvera les éléments communs et les notions propres à chaque système. Leur fréquentation est extrêmement précieuse pour acquérir la perspective globale et objective des systèmes juridiques, au lieu de s'en tenir aux quelques notions, brièvement esquissées, que contiennent les manuels de droit.

Les manuels d'initiation ou d'introduction au droit doivent faire partie de la bibliothèque du traducteur, quelle que soit sa spécialité. Les ouvrages de la collection «Que sais-je? 》 sont à recommander, à condition que leur lecture soit suivie de celle d'un traité (ou manuel) rédigé par une autorité incontestée dans le domaine.

25. L'excellent ouvrage d'Ernest Caparros et Jean Goulet, La documentation juridique (Québec, P.U.L., 1973, 182 p.) s'impose. 


\section{b) Les périodiques}

La doctrine s'exprime également par la voie d'articles, de critiques ou de commentaires publiés dans des revues et bulletins juridiques. L'utilité de ce type de documentation est indéniable. Un dictionnaire, un manuel, un répertoire sont périmés (en théorie) dès leur parution et il faut parfois attendre des années qu'une nouvelle édition vienne mettre à jour des notions qui vieillissent rapidement. Les périodiques, au contraire, suivent l'évolution du droit et de la pensée critique au mois, voire à la semaine près. Le traducteur y trouvera des termes et des notions qui ne figurent ni dans les manuels ni dans les dictionnaires. Le périodique est le reflet vivant de l'actualité juridique. Le traducteur ne peut pas se permettre de l'ignorer.

En résumé, étant donné que le traducteur devrait avoir à sa disposition les documents de base des trois domaines fondamentaux que sont la législation, la doctrine et la jurisprudence et qu'il lui est relativement aisé d'acquérir les deux premiers, il ne lui restera plus qu'à consulter les annuaires, répertoires, recueils de jurisprudence et autres rapports judiciaires dans les centres organisés pour recevoir cette volumineuse documentation. L'accès à la documentation juridique ne constitue qu'une des limites de la recherche documentaire.

\section{LES LIMITES DE LA RECHERCHE DOCUMENTAIRE}

Dans le domaine juridique, la recherche documentaire souffre de plusieurs handicaps qui freinent son développement et expliquent en partie la relative stagnation qu'a connue la lexicographie juridique. Ces handicaps sont de deux ordres. Les premiers découlent de la nature même du droit, des problèmes de classification qu'il pose dès que l'on franchit les frontières juridiques des États, et de la prolifération de la matière ; les seconds sont d'ordre linguistique et trahissent le faible intérêt des juristes pour leur langage.

Les perspectives ne sont pas toutes négatives et quelques points positifs permettent de penser qu'un certain équilibre pourrait être atteint dans un avenir prévisible.

\section{Les obstacles inhêrents au droit}

\section{Problèmes de classification}

Le chercheur qui aborde l'étude du droit en perspective comparée est d'emblée désorienté par les méthodes de classification des deux systèmes. Il ne verra pas toujours dans le système anglo-américain les grandes divisions générales et logiques des systèmes romano-germaniques, mais plutôt des découpages correspondant à des impératifs de procédure, ponctuels et concrets. Si l'on retrouve fréquemment dans les deux systèmes les notions parallèles de droit public et de droit privé, de substantive law (droit positif) et adjective law (la procédure), les documentalistes francophones, marqués par la tradition civiliste, classeront leurs unités documentaires par grandes catégories générales : 
- droit administratif

- droit civil

- droit commercial

- etc.

Leurs homologues anglophones, s'inspirant de considérations d'ordre pratique, suivront plutôt un ordre (ici théorique) comme celui-ci :

- Admiralty
- Antitrust
- Civil Rights
- Contracts
- Eminent domain

- Family Law

- Local Government

- Property

- Torts

Le profane est obligé d'établir lui-même les correspondances, ce qui n'est pas aussi évident que le juriste pourrait le croire. Toute comparaison devient délicate dès lors que les institutions de chaque système ne peuvent être comparées à celles de l'autre.

\section{La prolifération de la matière}

La recherche documentaire est aujourd'hui compliquée par la prolifération de la matière juridique, dont la diversité rend de surcroît le repérage problématique. On voit mal ce qui est juridique et ce qui ne l'est pas, le droit et sa terminologie couvrant un champ toujours plus vaste de l'activité humaine. Le droit administratif, la criminologie, le droit international (public mais également privé), entre autres, repoussent chaque jour davantage les frontières du * nondroit ». L'avènement de l'informatique juridique, tout en permettant au chercheur d'accéder plus rapidement à un certain type de documentation, obère le coût des recherches qui dépasse les ressources de la majorité des utilisateurs. Les centres de documentation juridique s'essoufflent à essayer de suivre un tel rythme.

Cette constatation conduit directement à l'éternel problème linguistique que pose la documentation juridique.

\section{Les obstacles linguistiques}

Une telle débauche d'activités et d'événements juridiques pose deux sortes de problèmes; le premier est d'ordre terminologique, le second, d'ordre lexicographique.

\section{Les problèmes terminologiques}

Cette question a déjà fait couler beaucoup d'encre ${ }^{26}$ et ses implications sont bien connues: absence d'un fonds terminologique essentiel; relativité des notions juridiques entraînant une polysémie déroutante pour le profane; carac-

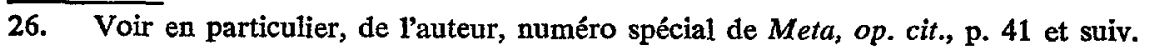


tère spécifique mais néanmoins imprécis de la terminologie d'un domaine en particulier; désaccord entre juristes même sur les divers sens possibles d'un terme et sur une définition commune à lui donner (ambiguilté perpétuée par le langage de la justice, pénale en particulier), etc.

Cette absence de stabilité sémantique est peut-être ce qui fait la force du droit, lui confère cette aura de mystère et ce prestige. La logique cartésienne, en mettant l'accent sur le * doute méthodique », éclaire la vision personnelle et intuitive de la réalité, mouvante et multiforme par excellence (voir les témoignages portant sur un même événement). Un excès de précision, des normes et des règles immuables et rigides sont contraires à l'esprit du droit, émanation synchronique des besoins et des préoccupations d'une société à un moment donné. Le droit ne peut fonctionner qu'à ce prix. Le meilleur exemple nous vient du droit international et de sa terminologie, délibérément floue pour laisser à chaque Etat souverain une certaine latitude flattant son esprit d'indépendance lorsqu'il interprète la norme internationale.

\section{Les limites de la lexicographie juridique}

Ces limites ont, elles aussi, été évoquées à maintes reprises : absence de productions lexicographiques normatives s'inspirant des grands dictionnaires établis par des lexicographes professionnels (étymologie, définitions, divers sens juridiques possibles, synonymie, antonymie, renvois...), dictionnaires de traductions rares et peu fiables (les solutions proposées ne sont souvent que des équivalents relevés dans des documents traduits); manque d'exhaustivité ou mélange des genres (trop de dictionnaires unilingues ou multilingues contiennent des termes dont le caractère juridique est très faible et relève plutôt du vocabulaire courant) ; vieillissement rapide de la terminologie, ce qui oblige à des mises à jour et à des remaniements fréquents (voir l'exemple du Lexique de termes juridiques, réédité tous les ans, comme le Petit Larousse).

Un tel bilan ne laisse pas entrevoir de solution miracle à brève échéance. Il est toutefois permis d'espérer car quelques indices positifs pourraient, s'ils se concrétisaient plus ou moins rapidement, rétablir un équilibre compromis.

\section{Les raisons d'espérer}

Depuis une dizaine d'années, de gros efforts ont été faits pour recenser les moyens et ressources en vue d'établir des centres de documentation juridique, des dictionnaires et encyclopédies répondant aux besoins des utilisateurs. La Communauté économique européenne, qui a de gros besoins dans ce domaine, a montré l'exemple. Le Canada, qui n'en a pas moins, n'est pas resté inactif. Peu de projets ont malheureusement abouti et il reste encore beaucoup à faire pour mener à terme les quelques projets entrepris. Le plus souvent, il faut le reconnaître, l'initiative reste individuelle, dispersée, répond à des préoccupations et à des besoins limités, nationaux ou régionaux. Le projet JURIVOC était porteur de grands espoirs, la mise en service de SOQUIJ rend d'indéniables services à la collectivité juridique. Le défaut de nombreux projets vient des objectifs parfois trop ambitieux. Il est des réussites plus discrètes, des recherches plus 
modestes qui pourraient inspirer des projets plus importants lorsque la «jurilinguistique » aura défini ses bases scientifiques avec rigueur et établi son statut de discipline autonome.

Plusieurs raisons permettent de croire en un avenir meilleur pour le traducteur juridique. Elles dépendent directement de l'évolution que suivra la jurilinguistique au cours des prochaines années et de son impact sur la lexicologie et la lexicographie juridiques.

\section{Le facteur lexicologique}

Les recherches en lexicologie appliquée au domaine du droit pourraient assurer une production lexicographique de qualité qui rendrait d'immenses services. Une recherche portant entre autres sur un \& vocabulaire général d'orientation juridique » s'inspirant du VGOS, serait opportune et aurait de nombreuses applications.

\section{Le facteur lexicographique}

Certaines productions lexicographiques, dans le domaine du droit, montrent la voie à suivre. Qui connaît et utilise les petits glossaires de l'Institut international de terminologie juridique et administrative sait de quoi nous parlons. Leur principal défaut est leur rareté, du moins dans les deux langues qui nous intéressent. Leur utilité n'en est pas moins grande.

Une autre raison d'espérer nous vient du groupe de jurilinguistes qui, à Ottawa, se sont attaqués au difficile problème des formulaires (législatifs) rédigés en français et devant servir de modèles de rédaction des lois et règlements. Le traducteur y puisera avec profit l'exemple dont il a besoin lorsqu'il traduit un texte anglais. Bien que l'on ne puisse enfermer le langage du droit dans un cadre formel, figé, de telles initiatives doivent être signalées et encouragées.

\section{CONCLUSION}

Dans le vécu quotidien du traducteur, la recherche documentaire occupe une place grandissante. En traduction juridique, cette place est souvent envahissante. Devant un tel volume de documentation, le traducteur éprouve parfois le sentiment bien compréhensible de l'aveugle devant l'éléphant : chaque partie du tout peut passer pour l'ensemble et être confondue avec lui. Or la recherche documentaire n'est qu'un élément permettant d'appréhender une réalité qui prend de nombreuses formes différentes et qui se situe bien au-delà de la simple connaissance de règles ou de normes. L'essentiel reste que le traducteur puisse se familiariser avec la question qui lui est posée et les outils s'y rapportant, non avec l'ensemble du droit, comme devrait le faire le juriste, qui est appelé à replacer les problèmes dans leur contexte global. Il doit néanmoins emprunter certaines méthodes et suivre dans certains cas le raisonnement de ce dernier afin de parvenir aux mêmes fins. Ce faisant, il prendra plus clairement conscience des limites de la recherche documentaire dans la spécialité qu'il a choisie. 
Que le traducteur et le terminologue soient préoccupés par les difficultés que présente la recherche documentaire en droit, cela se conçoit. Pourtant, ils ont d'aussi bonnes raisons d'être optimistes devant les progrès réalisés au cours des dix années écoulées et la volonté manifeste des milieux juridiques de chercher à sortir de l'ornière où ils se sont laissés enfoncer. L'exemple doit venir des juristes qui ne peuvent se contenter d'attendre que l'initiative linguistique vienne des traducteurs et des terminologues. Il faudra bien qu'ils unissent leurs efforts, d'ailleurs, s'ils veulent venir à bout des travaux d'Hercule que vient de leur imposer la Cour suprême dans son récent arrêt enjoignant le gouvernement du Manitoba de faire traduire en français les documents qu'il a produits depuis quelque... 90 ans. Dans une société qui a érigé le juridisme en vertu cardinale, le bon exemple ne pouvait venir que de la plus prestigieuse de ses institutions. Traducteurs, à vos marques! Prêts ? 